\title{
Using symbolic computation to prove nonexistence of distance-regular graphs
}

\author{
Janoš Vidali* \\ Faculty of Mathematics and Physics \\ University of Ljubljana, 1000 Ljubljana, Slovenia \\ janos.vidali@fmf.uni-lj.si
}

Submitted: Mar 30, 2018; Accepted: Sep 27, 2018; Published: Oct 19, 2018

(C) Janoš Vidali. Released under the CC BY license (International 4.0).

\begin{abstract}
A package for the Sage computer algebra system is developed for checking feasibility of a given intersection array for a distance-regular graph. We use this tool to show that there is no distance-regular graph with intersection array

$$
\begin{aligned}
& \{(2 r+1)(4 r+1)(4 t-1), 8 r(4 r t-r+2 t),(r+t)(4 r+1) ; \\
& 1,(r+t)(4 r+1), 4 r(2 r+1)(4 t-1)\} \quad(r, t \geqslant 1),
\end{aligned}
$$

$\{135,128,16 ; 1,16,120\},\{234,165,12 ; 1,30,198\}$ or $\{55,54,50,35,10 ; 1,5,20,45,55\}$. In all cases, the proofs rely on equality in the Krein condition, from which triple intersection numbers are determined. Further combinatorial arguments are then used to derive nonexistence.
\end{abstract}

Mathematics Subject Classifications: 05E30

\section{Introduction}

Distance-regular graphs were introduced around 1970 by N. Biggs [1]. As they are intimately linked to many other combinatorial objects, such as finite simple groups, finite geometries, and codes, a natural goal is trying to classify them.

Many distance-regular graphs are known, however constructing new ones has proved to be a difficult task. A number of feasibility conditions for distance-regular graphs have been found, which allows us to compile a list of feasible intersection arrays for small distance-regular graphs (or related structures, such as $Q$-polynomial association schemes), see Brouwer et al. [2, 3, 4] and Williford [22]. However, feasibility is no guarantee for

*This work is supported in part by the Slovenian Research Agency (research program P1-0285). 
existence, so proofs of nonexistence of distance-regular graphs with feasible intersection arrays are also a contribution to the classification. In certain cases, single intersection arrays have been ruled out $[12,13]$, while other proofs may show nonexistence for a whole infinite family of feasible intersection arrays $[6,9,19]$. In this paper we give proofs of nonexistence for distance-regular graphs belonging to a two-parameter infinite family, as well as for graphs with intersection arrays

$$
\begin{gathered}
\{135,128,16 ; 1,16,120\}, \\
\{234,165,12 ; 1,30,198\}, \\
\{55,54,50,35,10 ; 1,5,20,45,55\} .
\end{gathered}
$$

We develop a package called sage-drg [21] for the Sage computer algebra system [18]. Sage is free open-source software written in the Python programming language [17], with many functionalities deriving from other free open-source software, such as Maxima [16], which Sage uses for symbolic computation. The sage-drg package is thus also free opensource software available under the MIT license, written in the Python programming language, making use of the Sage library. The package can be used to check for feasibility of a given intersection array against known feasibility conditions, see Van Dam, Koolen and Tanaka for an up-to-date survey [7]. Furthermore, using equality in the Krein condition (see Theorem 1), restrictions on triple intersection numbers can be derived. In this paper, we use them to derive some nonexistence results. The sage-drg package also includes Jupyter notebooks demonstrating its use to obtain these results, as well as the notebook jupyter/Demo.ipynb giving some general examples of use of the package. A more detailed description of the sage-drg package is given in the supplementary files ${ }^{1}$.

The results from Sections 3, 4 and 6 appeared in the author's PhD thesis [20], where computation was done using a Mathematica [23] notebook originally developed by M. Urlep. Thus, the sage-drg package can be seen as a move from closed-source proprietary software to free open-source software, which allows one to check all code for correctness, thus making the results verifiable.

\section{Preliminaries}

In this section we review some basic definitions and concepts. See Brouwer, Cohen and Neumaier [3] for further details.

Let $\Gamma$ be a connected graph with diameter $d$ and $n$ vertices, and let $\partial(u, v)$ denote the distance between the vertices $u$ and $v$ of $\Gamma$. The graph $\Gamma$ is distance-regular if there exist constants $p_{i j}^{h}(0 \leqslant h, i, j \leqslant d)$, called the intersection numbers, such that for any pair of vertices $u, v$ at distance $h$, there are precisely $p_{i j}^{h}$ vertices at distances $i$ and $j$ from $u$ and $v$, respectively. In fact, all intersection numbers can be computed given only the intersection numbers $b_{i}=p_{1, i+1}^{i}$ and $c_{i+1}=p_{1, i}^{i+1}(0 \leqslant i \leqslant d-1)[3, \S 4.1 \mathrm{~A}]$. These intersection numbers are usually gathered in the intersection array $\left\{b_{0}, b_{1}, \ldots, b_{d-1} ; c_{1}, c_{2}, \ldots, c_{d}\right\}$. We also

\footnotetext{
${ }^{1}$ Available in the 'Appendix' at http://www.combinatorics.org/ojs/index.php/eljc/article/ view/v25i4p21
} 
define the valency $k=b_{0}$ and $a_{i}=k-b_{i}-c_{i}(0 \leqslant i \leqslant d)$, where $b_{d}=c_{0}=0$. A connected noncomplete strongly regular graph with parameters $(v, k, \lambda, \mu)$ is a distance-regular graph of diameter 2 with $v$ vertices, valency $k$ and intersection numbers $a_{1}=\lambda, c_{2}=\mu$.

Let $A_{i}(0 \leqslant i \leqslant d)$ be a binary square matrix indexed with the vertices of a graph $\Gamma$ of diameter $d$, with entry corresponding to vertices $u$ and $v$ equal to 1 precisely when $\partial(u, v)=i$. The matrix $A=A_{1}$ is the adjacency matrix of $\Gamma$. The graph $\Gamma$ is called primitive if all $A_{i}(1 \leqslant i \leqslant d)$ are adjacency matrices of connected graphs. A distanceregular graph of valency $k \geqslant 3$ that is not primitive is bipartite or antipodal (or both) [3, Thm. 4.2.1]. The spectrum of $\Gamma$ is defined to be the spectrum of $A$ (i.e., eigenvalues with multiplicities) and can be computed directly from the intersection array of $\Gamma[3, \S 4.1 \mathrm{~B}]$.

Suppose that $\Gamma$ is distance-regular. Let $\mathcal{M}$ be the Bose-Mesner algebra, i.e., the algebra generated by $A$. The matrices $\left\{A_{i}\right\}_{i=0}^{d}$ form a basis of $\mathcal{M}$, which also has a second basis $\left\{E_{i}\right\}_{i=0}^{d}$ consisting of projectors to the eigenspaces of $A[3, \S 2.2]$. Note that the indexing in this second basis depends on the ordering of eigenvalues. The descending ordering of eigenvalues is known as the natural ordering. We define the eigenmatrix $P$ and dual eigenmatrix $Q$ as $(d+1) \times(d+1)$ matrices such that $A_{j}=\sum_{i=0}^{d} P_{i j} E_{i}$ and $E_{j}=n^{-1} \sum_{i=0}^{d} Q_{i j} A_{i}$. The graph $\Gamma$ is called formally self-dual [3, p. 49] if $P=Q$ holds for some ordering of eigenvalues. The graph $\Gamma$ is called $Q$-polynomial $[3, \S 2.7]$ with respect to some ordering of eigenvalues if there exist real numbers $z_{0}, \ldots, z_{d}$ and polynomials $q_{j}$ of degree $j$ such that $Q_{i j}=q_{j}\left(z_{i}\right)(0 \leqslant i, j \leqslant d)$. Finally, we define the Krein parameters $q_{i j}^{h}[3, \S 2.3]$ as such numbers that $E_{i} \circ E_{j}=n^{-1} \sum_{h=0}^{d} q_{i j}^{h} E_{h}$, where o represents entrywise multiplication of matrices. A formally self-dual distance-regular graph is also $Q$-polynomial with respect to the corresponding ordering of eigenvalues and has $p_{i j}^{h}=q_{i j}^{h}(0 \leqslant i, j, h \leqslant d)$. In this paper, we will use the natural ordering for indexing, noting when a graph is $Q$-polynomial or formally self-dual for some other ordering.

For vertices $u, v, w$ of the distance-regular graph $\Gamma$ and integers $i, j, h(0 \leqslant i, j, h \leqslant d)$ we denote by $\left[\begin{array}{lll}u & v & w \\ i & j & h\end{array}\right]$ (or simply $\left[\begin{array}{lll}i & j & h\end{array}\right]$ when it is clear which triple $(u, v, w)$ we have in mind) the number of vertices of $\Gamma$ that are at distances $i, j, h$ from $u, v, w$, respectively. We call these numbers triple intersection numbers. They have first been studied in the case of strongly regular graphs [5], and later also for distance-regular graphs, see for example $[6,8,9,10,19]$. Unlike the intersection numbers, these numbers may depend on the particular choice of vertices $u, v, w$ and not only on their pairwise distances. We may however write down a system of $3 d^{2}$ linear Diophantine equations with $d^{3}$ triple intersection numbers as variables, thus relating them to the intersection numbers, cf. [9]:

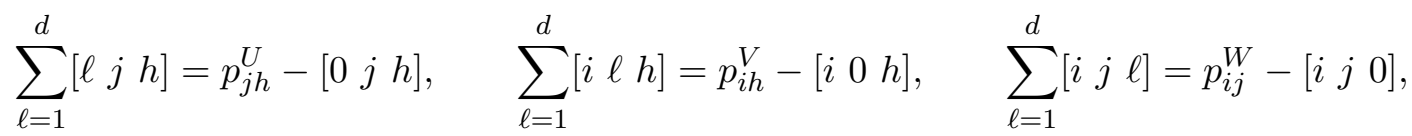

where $U=\partial(v, w), V=\partial(u, w), W=\partial(u, v)$, and

$$
\left[\begin{array}{lll}
0 & j & h
\end{array}\right]=\delta_{j W} \delta_{h V}, \quad\left[\begin{array}{lll}
i & 0 & h
\end{array}\right]=\delta_{i W} \delta_{h U}, \quad\left[\begin{array}{lll}
i & j & 0
\end{array}\right]=\delta_{i V} \delta_{j U} .
$$

Furthermore, we can use the triangle inequality to conclude that certain triple intersection numbers must be zero. Moreover, the following theorem sometimes gives additional equations. 
Theorem 1. ([6, Theorem 3], cf. [3, Theorem 2.3.2]) Let $\Gamma$ be a distance-regular graph with diameter $d$, dual eigenmatrix $Q$ and Krein parameters $q_{i j}^{h}(0 \leqslant i, j, h \leqslant d)$. Then,

$$
q_{i j}^{h}=0 \Longleftrightarrow \sum_{r, s, t=0}^{d} Q_{r i} Q_{s j} Q_{t h}\left[\begin{array}{lll}
u & v & w \\
r & s & t
\end{array}\right]=0 \quad \text { for all } u, v, w \in V \Gamma
$$

Together with integrality and nonnegativity of triple intersection numbers, we can use all of the above to either derive that the system of equations has no solution, or arrive at a small number of solutions, which gives us new information on the structure of the graph and may lead to proving its nonexistence.

\section{A two-parameter family of primitive graphs of diameter 3}

In [9], graphs meeting necessary conditions for the existence of extremal codes were studied. One of the families of primitive graphs of diameter 3 for which these conditions were met was

$$
\{a(p+1),(a+1) p, c ; 1, c, a p\},
$$

where $a=a_{3}, c=c_{2}$ and $p=p_{33}^{3}$. Graphs belonging to this family are $Q$-polynomial with respect to the natural ordering of eigenvalues precisely when the Krein parameter $q_{11}^{3}$ is zero, which is equivalent to

$$
c=\frac{1}{4}\left((p+1)^{2}+\frac{2 a(p+1)}{p+2}\right) .
$$

Hence, $p+2$ must divide $2 a$ for $c$ to be integral. If $p=2 r-1$, then $a=t(2 r+1)$ and $c=r(r+t)$ for some positive integers $r, t$, which gives us the two-parameter family

$$
\left\{2 r t(2 r+1),(2 r-1)(2 r t+t+1), r(r+t) ; 1, r(r+t), t\left(4 r^{2}-1\right)\right\} .
$$

In [9], nonexistence was shown for a feasible subfamily with $r=t \geqslant 2$. If, on the other hand, $p$ is even, integrality of the multiplicity of the second largest eigenvalue implies that we must have $p=4 r, a=(2 r+1)(4 t-1)$ and $c=(r+t)(4 r+1)$ for some positive integers $r, t$, giving the family

$$
\begin{array}{r}
\{(2 r+1)(4 r+1)(4 t-1), 8 r(4 r t-r+2 t),(r+t)(4 r+1) \\
1,(r+t)(4 r+1), 4 r(2 r+1)(4 t-1)\} .
\end{array}
$$

We find two one-parameter infinite subfamilies of feasible intersection arrays by setting $t=4 r^{2}$ or $t=4 r^{2}+2 r$ :

$$
\begin{gathered}
\left\{(2 r+1)(4 r+1)\left(16 r^{2}-1\right), 8 r^{2}\left(16 r^{2}+8 r-1\right), r(4 r+1)^{2} ;\right. \\
\left.1, r(4 r+1)^{2}, 4 r(2 r+1)\left(16 r^{2}-1\right)\right\}, \\
\left\{(2 r+1)(4 r+1)\left(16 r^{2}+8 r-1\right), 8 r^{2}(4 r+1)(4 r+3), r(4 r+1)(4 r+3) ;\right. \\
\left.1, r(4 r+1)(4 r+3), 4 r(2 r+1)\left(16 r^{2}+8 r-1\right)\right\} .
\end{gathered}
$$


There are also other feasible cases - for instance, when $r=2$, we have, besides the cases from the two subfamilies above, feasible examples when $t \in\{4,7,196\}$. The case with $r=1$ and $t=4$ belonging to the first subfamily above is also listed in the list of feasible parameter sets for 3-class $Q$-polynomial association schemes by J. S. Williford [22].

We now prove that a graph $\Delta$ with intersection array (4) does not exist. The proof parallels that of $[9$, Lems. 1, 3] - in fact, a significant part of the proof may be extended to the entire family (3), as it has been done in [20]. The computation needed to obtain the results in this section is illustrated in the jupyter/DRG-d3-2param.ipynb notebook included in the sage-drg package [21].

Lemma 2. Let $\Delta$ be a distance-regular graph with intersection array (4), and $u^{\prime}, v, w$ be vertices of $\Delta$ with $\partial\left(u^{\prime}, v\right)=1, \partial\left(u^{\prime}, w\right)=2$ and $\partial(v, w)=3$. Then $\left[\begin{array}{ccc}u^{\prime} & v & w \\ 3 & 3 & 3\end{array}\right]=1$.

Proof. Let $u$ be a vertex of $\Delta$ at distance 3 from both $v$ and $w$ (such a vertex exists since $\left.p_{33}^{3}=4 r>0\right)$. We consider the triple intersection numbers $[i j h]$ that correspond to $(u, v, w)$. As $q_{11}^{3}=q_{13}^{1}=q_{31}^{1}=0$, Theorem 1 gives three additional equations to the system (1), allowing us to express its solution in terms of a single parameter $\alpha=\left[\begin{array}{lll}3 & 3 & 3\end{array}\right]$. Let us express the counts of vertices at distance 1 or 2 from one of $u, v, w$ and at distance 3 from the other two vertices:

$$
\begin{aligned}
& {\left[\begin{array}{lll}
3 & 3 & 1
\end{array}\right]=\left[\begin{array}{lll}
3 & 1 & 3
\end{array}\right]=\left[\begin{array}{lll}
1 & 3 & 3
\end{array}\right]=\frac{(\alpha-4 r+1)(4 r+1)}{4 r-1},} \\
& {\left[\begin{array}{lll}
3 & 3 & 2
\end{array}\right]=\left[\begin{array}{lll}
3 & 2 & 3
\end{array}\right]=\left[\begin{array}{lll}
2 & 3 & 3
\end{array}\right]=\frac{8 r(4 r-1-\alpha)}{4 r-1} .}
\end{aligned}
$$

For the values above to be nonnegative, we must have $\alpha=4 r-1$, which means that they are all zero. As the choice of $u, v, w$ was arbitrary, this implies that any pair of vertices at distance 3 induces a set of $4 r+2$ vertices pairwise at distance 3 - in the terminology of [9], this is a maximal 1-code in $\Delta$. Since we have $a_{3} p_{33}^{3}=4 r(2 r+1)(4 t-1)=c_{3}$, it follows by [9, Prop. 2] that $\left[\begin{array}{ccc}u^{\prime} & v & w \\ 3 & 3 & 3\end{array}\right]=1$ holds.

Theorem 3. A distance-regular graph $\Delta$ with intersection array (4) does not exist.

Proof. Let $u^{\prime}, v, w$ be vertices of $\Delta$ with $\partial\left(u^{\prime}, v\right)=1, \partial\left(u^{\prime}, w\right)=2$ and $\partial(v, w)=3$ (such vertices exist, since we have $\left.p_{13}^{2}=b_{2}=(r+t)(4 r+1)>0\right)$. We consider the triple intersection numbers $[i j h]$ that correspond to $\left(u^{\prime}, v, w\right)$. By Lemma 2, we have $\left[\begin{array}{lll}3 & 3 & 3\end{array}\right]=1$. Using $q_{11}^{3}=0$, Theorem 1 gives an additional equation which allows us to obtain a unique solution to the system (1). However, we obtain $\left[\begin{array}{lll}1 & 1 & 3\end{array}\right]=2 t-1 / 2$, which is nonintegral for all integers $t$. Therefore, the graph $\Delta$ does not exist.

\section{A primitive graph with diameter 3 and 1360 vertices}

Let $\Lambda$ be a distance-regular graph with intersection array

$$
\{135,128,16 ; 1,16,120\} \text {. }
$$


This intersection array can be obtained from (2) by setting $a=15, c=16$ and $p=8$. The graph $\Lambda$ has diameter 3 and 1360 vertices. It is not $Q$-polynomial, however its Krein parameter $q_{33}^{3}$ is zero. We show that such a graph does not exist. The computation needed to prove Theorem 4 is illustrated in the jupyter/DRG-135-128-16-1-16-120. ipynb notebook included in the sage-drg package [21].

Theorem 4. A distance-regular graph $\Lambda$ with intersection array (5) does not exist.

Proof. Let $u, v, w$ be three pairwise adjacent vertices of $\Lambda$ (such vertices exist, since we have $\left.p_{11}^{1}=6>0\right)$. We consider triple intersection numbers $[i j h]$ that correspond to $(u, v, w)$. As $q_{33}^{3}=0$, Theorem 1 gives an additional equation to the system (1), allowing us to express its solution in terms of a single parameter $\alpha=\left[\begin{array}{lll}1 & 1 & 1\end{array}\right]$. In particular, we obtain

$$
\left[\begin{array}{lll}
3 & 3 & 3
\end{array}\right]=\frac{71-27 \alpha}{8} .
$$

Clearly, $\alpha$ must be a nonnegative integer. For [ $\left[\begin{array}{lll}3 & 3 & 3\end{array}\right]$ to be nonnegative, we must have $\alpha \in\{0,1,2\}$. However, [ [ $\left.\begin{array}{lll}3 & 3 & 3\end{array}\right]$ is still nonintegral in these cases, showing that the graph $\Lambda$ does not exist.

\section{A primitive graph with diameter 3 and 1600 vertices}

Let $\Xi$ be a distance-regular graph with intersection array

$$
\{234,165,12 ; 1,30,198\} \text {. }
$$

The graph $\Xi$ has diameter 3 and 1600 vertices. The intersection array (6) has been found as an example of a feasible parameter set for a distance-regular graph which is formally self-dual for an ordering of eigenvalues distinct from the natural ordering - in fact, $\Xi$ is $Q$-polynomial for the ordering $0,2,3,1$, so its Krein parameters $q_{22}^{1}, q_{12}^{2}$ and $q_{21}^{2}$ are zero. The intersection array (6) is also listed in the list of feasible parameter sets for 3-class $Q$-polynomial association schemes by J. S. Williford [22]. We show that such a graph does not exist. The computation needed to prove Theorem 5 is illustrated in the jupyter/DRG-234-165-12-1-30-198. ipynb notebook included in the sage-drg package [21].

Theorem 5. A distance-regular graph $\Xi$ with intersection array (6) does not exist.

Proof. Let $u, v, w$ be three vertices of $\Xi$ that are pairwise at distance 3 (such vertices exist, since we have $p_{33}^{3}=8>0$ ). We consider triple intersection numbers $[i j h]$ that correspond to $(u, v, w)$. As $q_{22}^{1}=q_{12}^{2}=q_{21}^{2}=0$, Theorem 1 gives three additional equations to the system (1), allowing us to express its solution in terms of a single parameter $\alpha=\left[\begin{array}{llll}3 & 3 & 3\end{array}\right]$. In particular, we obtain

$$
\left[\begin{array}{lll}
3 & 3 & 2
\end{array}\right]=\left[\begin{array}{lll}
3 & 2 & 3
\end{array}\right]=\left[\begin{array}{lll}
2 & 3 & 3
\end{array}\right]=-17-4 \alpha .
$$

Clearly, $\alpha$ must be nonnegative, but then we have $\left[\begin{array}{lll}3 & 3 & 2\end{array}\right]=\left[\begin{array}{lll}3 & 2 & 3\end{array}\right]=\left[\begin{array}{lll}2 & 3 & 3\end{array}\right]<0$, a contradiction. We conclude that the graph $\Xi$ does not exist. 


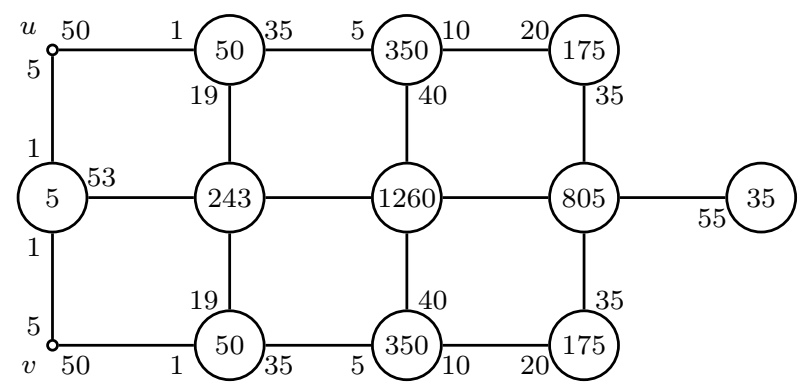

Figure 1: The partition of vertices of $\Sigma$ by distance from a pair of vertices $u, v$ at distance 2. The part that is at distance $i$ from $u$ and distance $j$ from $v$ has size $p_{i j}^{2}$. As the graph is bipartite, the intersection number $p_{i j}^{2}$ is nonzero only when $i+j$ is even. Moreover, there are no edges within each part. It is natural to consider [ $\left[\begin{array}{lll}1 & 1 & 1\end{array}\right]$ for $w$ at distance 2 from both $u$ and $v$, see Lemma 6 .

\section{A bipartite graph with diameter 5}

Let $\Sigma$ be a distance-regular graph with intersection array

$$
\{55,54,50,35,10 ; 1,5,20,45,55\} \text {. }
$$

This intersection array appears in the list of feasible intersection arrays for bipartite nonantipodal distance-regular graphs of diameter 5 by Brouwer et. al. [3, p. 418] as an open case. The existence of such a graph would give a counterexample to a conjecture by MacLean and Terwilliger [15], cf. Lang [14]. The computation needed to obtain the results in this section is illustrated in the jupyter/DRG-55-54-50-35-10-bipartite.ipynb notebook included in the sage-drg package [21].

The graph $\Sigma$ has diameter 5 and 3500 vertices. The partition of $\Sigma$ corresponding to two vertices at distance 2 is shown in Figure 1 . The graph is $Q$-polynomial for the natural ordering of eigenvalues, see for example [3, p. 418]. Moreover, as the graph is bipartite, it is also $Q$-antipodal [3, Thm. 8.2.1]. Many Krein parameters are zero, in particular $q_{11}^{3}$ and $q_{11}^{4}$ due to the triangle inequality. We use this fact in the proof of the following statement.

Lemma 6. Let $\Sigma$ be a distance-regular graph with intersection array (7), and $u, v, w$ be vertices of $\Sigma$ that are pairwise at distance 2 . Then $\left[\begin{array}{lll}u & v & w \\ 1 & 1 & 1\end{array}\right] \leqslant 1$.

Proof. We consider the triple intersection numbers $\left[\begin{array}{lll}i & j & h\end{array}\right]$ that correspond to $(u, v, w)$. Since the graph $\Sigma$ is bipartite, we have $[i j h]=0$ whenever any of the sums $i+j, j+h$, $h+i$ is odd. As $q_{11}^{3}=q_{11}^{4}=0$, Theorem 1 gives us two additional equations to the system (1), thus allowing us to express the solution of the system in terms of a single parameter $\alpha=\left[\begin{array}{lll}1 & 1 & 1\end{array}\right]$. In particular, we obtain

$$
\left[\begin{array}{lll}
5 & 5 & 5
\end{array}\right]=20-12 \alpha .
$$

The integrality and nonnegativity of $\left[\begin{array}{lll}5 & 5 & 5\end{array}\right]$ now gives $\alpha \leqslant\lfloor 5 / 3\rfloor=1$. 
Note. It can also be shown with a method similar to the one used in Lemma 6 that the graph $\left[\Sigma_{5}(u)\right]_{2}$ for a vertex $u \in V \Sigma$ (i.e., the graph of vertices at distance 5 from a vertex $u$, with adjacency corresponding to distance 2 in $\Sigma$ ) is strongly regular with parameters $(v, k, \lambda, \mu)=(210,99,48,45)$. A strongly regular graph with such parameters has been constructed by M. Klin [11].

Theorem 7. A distance-regular graph $\Sigma$ with intersection array (7) does not exist.

Proof. Let $u$ and $v$ be vertices of $\Sigma$ at distance 2, see Figure 1, and let $\{i j\}$ denote the set of vertices at distances $i$ and $j$ from $u$ and $v$, respectively. There are $p_{11}^{2}(k-2)=$ $5 \cdot 53=265$ edges between the sets $\left\{\begin{array}{ll}1 & 1\end{array}\right\}$ and $\{22\}$. However, the cardinality of the latter set is $p_{22}^{2}=243<265$, so there is a vertex $w \in\{22\}$ that has at least two neighbours in $\left\{\begin{array}{ll}1 & 1\end{array}\right\}$, i.e., $\left[\begin{array}{lll}u & v & w \\ 1 & 1 & 1\end{array}\right] \geqslant 2$, which is in contradiction with Lemma 6. Hence, the graph $\Sigma$ does not exist.

\section{Acknowledgements}

I would like to thank Michael Lang for bringing the intersection array (7) to my attention and for noticing some bugs and proposing new functionality for the sage-drg package.

\section{References}

[1] N. L. Biggs. Intersection matrices for linear graphs. In D. J. A. Welsh, editor, Combinatorial Mathematics and its applications, pages 15-23. Academic Press, London, 1971.

[2] A. E. Brouwer. Parameters of distance-regular graphs, 2011. http://www .win.tue. $\mathrm{nl} / \sim \mathrm{aeb} / \mathrm{drg} /$ drgtables.html.

[3] A. E. Brouwer, A. M. Cohen, and A. Neumaier. Distance-regular graphs, volume 18 of Ergebnisse der Mathematik und ihrer Grenzgebiete (3) [Results in Mathematics and Related Areas (3)]. Springer-Verlag, Berlin, 1989. doi:10.1007/978-3-642-74341-2.

[4] A. E. Brouwer, A. M. Cohen, and A. Neumaier. Corrections and additions to the book 'Distance-regular graphs', 1994. http://www.win.tue.nl/ aeb/drg/.

[5] P. J. Cameron, J.-M. Goethals, and J. J. Seidel. Strongly regular graphs having strongly regular subconstituents. J. Algebra, 55(2):257-280, 1978. doi : 10.1016/0021-8693(78)90220-X.

[6] K. Coolsaet and A. Jurišić. Using equality in the Krein conditions to prove nonexistence of certain distance-regular graphs. J. Combin. Theory Ser. A, 115(6):1086-1095, 2008. doi:10.1016/j.jcta.2007.12.001. 
[7] E. R. van Dam, J. H. Koolen, and H. Tanaka. Distance-regular graphs. Electron. J. Combin., DS:22, 2016. http://www.combinatorics.org/ojs/index.php/eljc/ article/view/DS22.

[8] A. Jurišić, J. Koolen, and P. Terwilliger. Tight distance-regular graphs. J. Algebraic Combin., 12(2):163-197, 2000. doi:10.1023/A:1026544111089.

[9] A. Jurišić and J. Vidali. Extremal 1-codes in distance-regular graphs of diameter 3. Des. Codes Cryptogr., 65(1-2):29-47, 2012. doi:10.1007/s10623-012-9651-0.

[10] A. Jurišić and J. Vidali. Restrictions on classical distance-regular graphs. J. Algebraic Combin., 46(3-4):571-588, 2017. doi:10.1007/s10801-017-0765-3.

[11] M. Klin, C. Pech, S. Reichard, A. Woldar, and M. Ziv-Av. Examples of computer experimentation in algebraic combinatorics. Ars Math. Contemp., 3(2):238-248, 2010. http://amc-journal.eu/index.php/amc/article/view/119.

[12] J. H. Koolen. A new condition for distance-regular graphs. European J. Combin., 13(1):63-64, 1992. doi:10.1016/0195-6698(92)90068-B.

[13] E. Lambeck. Some elementary inequalities for distance-regular graphs. European J. Combin., 14(1):53-54, 1993. doi:10.1006/eujc.1993.1008.

[14] M. S. Lang. Bipartite distance-regular graphs: the $Q$-polynomial property and pseudo primitive idempotents. Discrete Math., 331:27-35, 2014. doi:10.1016/j.disc.2014.04.025.

[15] M. S. MacLean and P. Terwilliger. Taut distance-regular graphs and the subconstituent algebra. Discrete Math., 306(15):1694-1721, 2006. doi:10.1016/j.disc.2006.03.046.

[16] Maxima. Maxima, a computer algebra system. version 5.39.0, 2017. http://maxima. sourceforge.net/.

[17] Python Software Foundation. Python Language Reference, version 2.7.13, 2017. http://www . python.org.

[18] The Sage Developers. SageMath, the Sage Mathematics Software System (Version 7.6), 2017. http://www. sagemath.org.

[19] M. Urlep. Triple intersection numbers of $Q$-polynomial distance-regular graphs. European J. Combin., 33(6):1246-1252, 2012. doi:10.1016/j.ejc.2012.02.005.

[20] J. Vidali. Codes in distance-regular graphs. PhD thesis, University of Ljubljana, 2013. http://eprints.fri.uni-lj.si/2167/(in Slovene).

[21] J. Vidali. jaanos/sage-drg: sage-drg Sage package v0.8, 2018. https://github. com/jaanos/sage-drg/, doi:10.5281/zenodo.1418410. 
[22] J. S. Williford. Primitive 3-class Q-polynomial association schemes, 2017. Mirrored version available at https://jaanos.github.io/tables/qpoly/qprim3_table. html.

[23] Wolfram Research, Inc. Mathematica, Version 8.0. Champaign, Illinois, 2010. http: //www. wolfram.com/mathematica/. 